04

\title{
Два режима автомодельной эволюции заряженной плазмы
}

\author{
(C) B.A. Павлов \\ Санкт-Петербургский государственный университет, физический фракультет, \\ 198504 Санкт-Петербург, Петергоф, Россия \\ e-mail: v.pavlov@spbu.ru
}

(Поступило в Редакцию 22 апреля 2017 г.)

Получено аналитическое описание двух автомодельных режимов эволюции сильноточного пучка заряженных частиц. Рассматриваемая ситуация относится к разделу электродинамики заряженной плазмы (nonneutral plasma). Использовано описание процесса на основе нелинейной одномерной эволюции плотности заряда $w(x, t)$ в канале длинной линии с нелинейными распределенными параметрами - сопротивлением, емкостью и индуктивностью: $R=R(w), C=C(w), L=0$. Показано, что на начальном этапе происходит ускорение фронта $w(x, t)$. На втором этапе происходит торможение его. За основу описания процесса в канале взят закон сохранения зарядов. Применен „кинематический“ подход, заключающийся в идеализации, когда уравнение для двух неизвестных функций: плотность заряда и плотность тока в канале приводится к уравнению для одной неизвестной функции $w(x, t)$. Исследована ситуация сильно нелинейного волнового процесса. Построено разрывное решение $w(x, t)$ с нулевым граничным условием на бесконечности. Использование такого модельного описания может претендовать только на выявление основных качественных закономерностей сложного процесса. Получены аналитические законы эволюции скорости фронта и длины возмущенной области. На основе анализа экспериментальных данных об эволюции стримеров предложена взаимосвязь параметра, характеризующего нелинейность процесса с величиной заряда в междуэлектродном промежутке.

DOI: $10.21883 /$ JTF.2018.03.45590.2306

\section{Введение}

Одним из примеров заряженной сильноточной плазмы являются стримеры длинных искровых разрядов. Процессы в стримере искрового разряда настолько сложны, что до сих пор отсутствуют физически прозрачные и надежные формулы зависимости скорости стримера от определяющих параметров. Сегодня нет ни адекватной теории, ни адекватных численных расчетов эволюции стримера [1]. Имеется только упрощенное, качественное понимание закономерности такой эволюции [1-18].

Типичные значения параметров стримера изменяются значительно [1]. В воздухе скорость стримера достигает $\left(10^{5}-10^{7}\right) \mathrm{ms}^{-1}$. В окрестности фронта создается плазма с концентрацией электронов до значения $10^{20} \mathrm{~m}^{-3}$. В лабораторных условиях ток в канале порядка $1 \mathrm{~A}$. Для молнии этот ток на два порядка больше. У молнии потенциал в окрестности фронта на 1-2 порядка больше, чем максимально достижимый потенциал в лабораторных экспериментах.

\section{Постановка задачи}

Рассмотрим сильно идеализированную одномерную задачу. Пусть на самой начальной стадии искрового разряда однородный электронейтральный газ еще неподвижен. Сильноточный пучок заряженных частиц исследуем как следствие мгновенного выделения заряда $2 Q=$ const в начальный момент $t=0$ на плоскости, перпендикулярной оси $x$ и проходящей через точку $x=0$. Действие такого источника сопровождается возникновением плотности заряда $w(x, t)$ и плотности тока $j(x, t)$, которые описываем законом сохранения заряда

$$
\begin{gathered}
\frac{\partial w}{\partial t}+\frac{\partial}{\partial x} j=0 \\
\int_{0}^{\infty} w(x, 0) d x=Q .
\end{gathered}
$$

Использование (1), (2) является большой идеализацией явления. В частности, при этом не учитывается возможность нарушения закона $Q=$ const coхранения заряда за счет наличия проводящих ток плазменных „мостиков“, реализующих потери зарядов в канале при протекании реакций ионизации и диссоциации. Ограничимся рассмотрением потенциального электрического поля

$$
E=-\frac{\partial}{\partial x} U(x, t), \quad U(\infty, t)=0 .
$$

Используем простейшую нелинейную форму закона Ома для медленных процессов

$$
j=j(U)=-\frac{1}{R(U)} \frac{\partial}{\partial x} U,
$$

где $R(U)$ - нелинейное погонное сопротивление канала. Понятие нелинейной погонной емкости канала $C(U)$ введем на основе определения

$$
C(U) \equiv \frac{d w}{d U}
$$

где использована идеализация $w=w(U(x, t))$. 
На основе (3), (4) имеем представление для плотности тока

$$
j=j(w)=-\frac{1}{R(w)} \frac{d U}{d w} \frac{\partial}{\partial x} w=-\frac{1}{R(w) C(w)} \frac{\partial}{\partial x} w .
$$

В такой идеализации закон сохранения заряда (1) для двух неизвестных функций $w, j$ принимает вид уравнения нелинейной одномерной диффузии для одной функции $w$, описывающий эволюцию $w(x, t)$ в длинной линии с распределенными параметрами:

$$
\begin{gathered}
\frac{\partial w}{\partial t}+\frac{\partial}{\partial x}\left[-\frac{1}{T(w)} \frac{\partial}{\partial x} w\right]=0, \\
T(w) \equiv R(w) C(w), \quad w=w(j(x, t)) .
\end{gathered}
$$

Использована идеализация существования связи функций $w=w(j(x, t))$. Такой прием использовался Дж. Уиземом $[19,20]$ для исследования качественных закономерностей формирования и эволюции ударной волны в нейтральном газе. Такая процедура названа [19] „кинематическим приближением“ для уравнения неразрывности. По сути, идея этого приближения аналогична способу получения описания „простой волны Римана“ при решении гиперболической системы уравнений. При получении „кинематического приближения“ отсутствует необходимость гиперболичности системы уравнений.

Возможно более строгое (более точное) описание, когда функция $T(x, t)$ считается неизвестной функцией. При этом в дополнении к (5) необходимо использовать дополнительные уравнения материальной связи, которые устанавливают достаточно адекватное соотношение между функциями, $T$ и $w$. При этом реализуется нелинейное самосогласованное описание. Еще возможно альтернативное описание, имеющее более узкую область применимости, когда для нахождения функции $T(x, t)$ используется самостоятельная, независимая от уравнения (5) система уравнений. В последнем случае возникает не самосогласованное описание с использованием линейной эволюции на основе (5) с коэффициентом $T(x, t)$, зависящим от координат и времени.

Широко используется [1-3] моделирование процессов в стримерном канале на основе линейных уравнений длинной линии с распределенными параметрами, зависящими от координаты и времени $R_{0}=R_{0}(t, x)$ - линейное погонное сопротивление канала, $C_{0}=C_{0}(t, x)-$ линейная погонная емкость канала, $L_{0}=0-$ линейная погонная индуктивность канала. Такое приближение соответствует замене $R \rightarrow R_{0}, C \rightarrow C_{0}$ в уравнении кинематического описания эволюции (5).

Используем степенную аппроксимацию:

$$
T(w)=T_{0}\left(\frac{w_{0}}{w}\right)^{n}, \quad T_{0}=\text { const, } \quad w_{0}=\text { const. }
$$

Здесь $n-$ произвольное число, значение параметра $n$ находится из экспериментальных данных. Уравнение (5) принимает вид

$$
\frac{\partial w}{\partial t}-\kappa \frac{\partial^{2} w^{n+1}}{\partial x^{2}}=0, \quad \kappa \equiv \frac{1}{(n+1) T_{0} w_{0}^{n+1}} .
$$

Плотность тока $j(w)$ описывается нелинейным законом Ома (соотношением Фурье-Ньютона):

$$
j(w)=-\frac{\kappa}{n+1} \frac{\partial w^{n+1}}{\partial x} .
$$

Решение уравнения (7) построим при начальном условии и условии на бесконечности:

$$
\begin{gathered}
\int_{0}^{\infty} w(x, 0) d x=Q, \quad Q=\text { const, } \\
\left.w(\infty, t)\right|_{t>0}=0,\left.\quad w(x, t)\right|_{\substack{t<0 \\
x \neq 0}}=0 .
\end{gathered}
$$

Анализ показывает отсутствие непрерывных решений $w(x, t)$. Возможно удовлетворение условия на бесконечности $\left.w(\infty, t)\right|_{t>0}=0$, если ввести понятие разрывных решений.

В нашей работе [21] рассмотрение эволюции стримера ограничивалось только автомодельным режимом торможения фронта стримера. Аналогом задачи (7)-(9) является исследование процессов другой физической природы: нелинейного распространения тепла при теплопроводности, зависящей от температуры [22], и исследование фильтрации жидкости и газа в пористой среде [23,24].

\section{Теоретический анализ}

В рассматриваемой идеализированной задаче имеется четыре размерных определяющих параметра: $t, x, Q, \kappa$ и один безразмерный параметр $n$. Согласно П-теореме теории размерности, плотность заряда $w$ можно представить в виде автомодельного решения первого рода в виде

$$
w=\left(\frac{Q^{2}}{t \kappa}\right)^{\frac{1}{n+2}} \Phi(\Pi),
$$

где $\Phi(П)$ - безразмерная функция, зависящая от безразмерного параметра П:

$$
\Pi=x\left(\frac{1}{t \kappa Q^{n}}\right)^{\frac{1}{n+2}} .
$$

Не получая вида решения для функции $\Phi(П)$, можно сделать вывод - в рамках используемой идеализации функциональные представления полей оказываются одинаковыми для ситуаций различного знака заряда: $Q>0$, $Q<0$. Заряженная плазма, перемещающаяся в сторону анода и в сторону катода, описывается одинаковыми функциями. Отличие этих двух ситуаций характеризуется различным набором параметров $t, x, Q, n$.

На основе (7), (10) получается нелинейное обыкновенное дифференциальное уравнение для функции $\Phi(\Pi)$ :

$$
\frac{d^{2}}{d \Pi^{2}} \Phi^{n+1}+\frac{\Pi}{n+2} \frac{d}{d \Pi} \Phi+\frac{1}{n+2} \Phi=0 .
$$


Уравнение (12) не имеет вещественного непрерывного решения с непрерывными производными, удовлетворяющего нулевому условию на бесконечности: $\Phi(\infty, t)=0$. В области $\Pi^{2} \leq \Pi_{0}^{2}$ уравнение (12) удовлетворяется вещественной непрерывной функцией вида

$$
\Phi=\text { const }\left(\Pi_{0}^{2}-\Pi^{2}\right)^{\alpha}, \quad \Pi^{2} \leq \Pi_{0}^{2} .
$$

Требование $\Phi(\infty, t)=0$ можно обеспечить использованием разрывного решения при выполнении условия $\Phi\left(\Pi^{2}\right)=0$ в области $\Pi^{2} \geq \Pi_{0}^{2}=$ const. Параметры const и $\alpha$ находятся при подстановке (13) в уравнение (12). Имеет место разрывное решение

$$
\Phi= \begin{cases}{\left[\frac{N(n)}{2}\left(\Pi_{0}^{2}-\Pi^{2}\right)\right]^{\frac{1}{n}},} & N(n) \equiv \frac{n}{(n+1)(n+2)}, \\ 0, & \Pi^{2} \leq \Pi_{0}^{2} \\ & \Pi^{2} \geq \Pi_{0}^{2} .\end{cases}
$$

Конкретное значение параметра $\Pi_{0}$ находится из условия сохранения (2) заряда в канале. Это условие приводится к равенству, которое справедливо при любом значении времени $t$ :

$$
\int_{0}^{\infty} w(x, t) d x=Q,
$$

это соотношение эквивалентно уравнению для нахождения параметра $\Pi_{0}$

$$
\begin{gathered}
\int_{0}^{\infty} \Phi(\Pi) d \Pi=\left[\frac{N(n)}{2}\right]^{\frac{1}{n}} \int_{0}^{\Pi_{0}}\left(\Pi_{0}^{2}-\Pi^{2}\right)^{\frac{1}{n}} d \Pi=1, \\
N(n) \equiv \frac{n}{(n+1)(n+2)},
\end{gathered}
$$

получаем представление для параметра П 0 в виде

$$
\Pi_{0}=\Pi_{0}(n)=(n+2)^{\frac{n+1}{n+2}}\left\{2 \frac{n+1}{n}\left[\frac{\Gamma\left(\frac{1}{n}+\frac{1}{2}\right)}{\sqrt{\pi} \Gamma\left(\frac{1}{n}\right)}\right]^{n}\right\}^{\frac{1}{n+2}}
$$

где Г — гамма-функция.

Таким образом, автомодельное решение (13) построено. Такое решение уравнения (12) с учетом нулевого значения на бесконечности и обладающее свойством сохранения (9) впервые было получено в работе о распространении тепла, при теплопроводности, зависящей от температуры [22]. В математическом отношении рассматриваемая нами задача эквивалентна исследованиям в работах [21-24]. Вещественность функции $\Phi(П)$ обеспечивается выполнением условия на безразмерный параметр $n$ :

$$
N(n) \equiv \frac{n}{(n+1)(n+2)}>0 .
$$

Следствием (15) является одно из возможных ограничений на безразмерный параметр $n=n_{1,2}$ :

$$
-2<n_{1}<-1, \quad \text { либо } \quad n_{2}>0 \text {. }
$$

Очень ,жесткая“ постановка задачи (мгновенное выделение заряда $Q$ в точке $x=0)$ привела к упрощению рассмотрения задачи за счет отсутствия временного и пространственного масштабов. Это позволило построить автомодельное решение первого рода.

Параметр $\Pi_{0}$ характеризует положение фронта $x_{0}(t)$ :

$$
\begin{gathered}
w\left(x_{0}(t), t\right)=\left.0 \quad \Pi\right|_{x_{0}(t)}=\Pi_{0}, \\
x_{0}(t)=t^{\frac{1}{n+2}}\left[\Pi_{0}\left(\kappa Q^{n}\right)^{\frac{1}{n+2}}\right] .
\end{gathered}
$$

Нетривиальным обстоятельством является тот факт, что параметр $\Pi_{0}$ зависит от значения безразмерного числа $n$. Для описания двух автомодельных режимов эволюции заряженной плазмы существуют два значения параметра $\Pi_{0}$ :

$$
\Pi_{01} \equiv \Pi_{0}\left(n_{1}\right), \quad n_{1}<0, \quad \Pi_{02} \equiv \Pi_{0}\left(n_{2}\right), \quad n_{2}>0 .
$$

Индекс 1 относится к режиму ускорения фронта стримера, а индекс 2 относится к режиму торможения фронта стримера.

Автомодельная нелинейная волна диффузии плотности заряда, удовлетворяющая нулевому условию на бесконечности, может быть описана разрывной функцией:

$$
\begin{array}{ll}
w(x, t)=w(0, t)\left[1-\frac{x^{2}}{x_{0}^{2}(t)}\right]^{\frac{1}{n}}, & x^{2} \leq x_{0}^{2}(t), \\
w(x, t)=0 & x^{2} \geq x_{0}^{2}(t) .
\end{array}
$$

Скорость перемещения фронта описывается соотношениями

$$
\begin{gathered}
V_{0}(t)=\frac{d x_{0}}{d t}=\frac{1}{n+2} \frac{x_{0}}{t}, \quad V_{0}(t) \sim t^{\frac{n+1}{n+2}}, \quad V_{0} \sim Q_{0}^{\frac{n}{n+2}}, \\
x_{0}(t)=x_{0}(t)=t^{\frac{1}{n+2}}\left[\Pi_{0}\left(\kappa Q^{n}\right)^{\frac{1}{n+2}}\right]=(\text { const }) \cdot t^{\frac{1}{n+2}} .
\end{gathered}
$$

В рамках принятой модели при выполнении условий (15), (16) возможны два режима автомодельной эволюции: Режим № $1 n=n_{1}<0$ : только ускорение фронта возмущенной области заряженной плазмы. Такой режим эволюции стримера исследовался экспериментально с помощью скоростной фотосъемки [18]. Режим № $2 n=n_{2}>0$ : только торможение фронта возмущенной области заряженной плазмы исследовался в работах $[2-17,21]$.

В случае $n=n_{1}$, где параметр $n_{1}<0$ удовлетворяет неравенствам $-2<n_{1}<-1$, имеет место бесконечный скачок плотности заряда $w$ в точке $\Pi_{01}$ на фронте $x_{01}(t)$. Такая ситуация не анализировалась в нашей работе [21].

Авторами [22] ошибочно указано существование вещественного автомодельного решения при выполнении 
условия $-2<n_{1}<0$ вместо условия $-2<n_{1}<-1$. По этой причине в [22] не отмечено отсутствие автомодельного режима ускорения в диапазоне параметра $-1<n_{1}<0$, и не отмечено отсутствие автомодельного линейного режима ускорения при условии $\left|n_{1}\right| \ll 1$. Последнее свойство режима ускорения возмущенной области является проявлением достаточно редкой аномальности слабо нелинейных волновых процессов.

Физически реализуемая ситуация соответствует непрерывному описанию плотности тока (9) в окрестности фронта возмущенной области $x=x_{0}$. Исследуем условие непрерывности плотности тока на фронте $x=x_{0}$ при перемещении фронта в область положительных значений: $x>0$. Скачок $[j]$ плотности тока описывается соотношением

$$
[j] \sim\left[\frac{\partial w^{n+1}}{\partial x}\right]=\left.w^{n} \frac{\partial w}{\partial x}\right|_{x_{0}-0}-\left.w^{n} \frac{\partial w}{\partial x}\right|_{x_{0}+0} .
$$

Перед фронтом выполняется условие $\left.\left(w^{n} \frac{\partial w}{\partial x}\right)\right|_{x_{0}+0}=$ $=0$, это обеспечивает нулевые условия на бесконечности. Непрерывность плотности тока обеспечивается за счет выполнении условия позади фронта при любом знаке параметра $n$ :

$$
\left.w^{n} \frac{\partial w}{\partial x}\right|_{x_{0}-0}=0,\left.\left.\quad w^{n}\right|_{x_{0}-0} \sim\left(1-\frac{x^{2}}{x_{0}^{2}}\right)\right|_{x_{0}-0}=0 .
$$

В режиме торможения $n=n_{2}$ на фронте допустимо разрывное изменение производной $\frac{\partial w}{\partial x}$. Величина этого разрыва может быть даже бесконечной. В режиме ускорения $n=n_{1}$ на фронте имеется бесконечный скачок плотности заряда $w$. Таким образом, плотность тока в окрестности фронта возмущенной области $x=x_{0}$ непрерывна при любом режиме автомодельной эволюции стримера.

На стадии ускорения выполняются условия $-2<n_{1}<$ $<-1$, реализуется эффект сильной нелинейности. Аномальной особенностью используемой идеализации режима ускорения является то, что решение (17) не имеет предельного перехода к линейному приближению.

Так как $x_{0}(t) \propto t^{1 / n+2}$, то в рамках используемой модели при условии $n \rightarrow-2$ имеется второе проявление аномальности: происходит мгновенное перемещение фронта на бесконечное расстояние. В ситуации, близкой к выполнению условия $n \rightarrow-2$, необходим учет дополнительных факторов.

Режим торможения $n=n_{2}>0$ реализуется при выполнении условия $n_{2}>0$, при этом не происходит остановки фронта на конечных расстояниях. Следует отметить, что при выполнении условия $n>1$ на фронте формируется „слабый разрыв“ функции $w$. В такой волне отсутствует скачок функции $w$ и формируется скачок производной, возникает „излом“ функции $\Phi(П)$ на фронте в точке $\Pi_{02}$. Выполнение условия (15) обеспечивает увеличение размера возмущенной области как на стадии ускорения, так и на стадии торможения фронта.
Поэтапное использование двух идеализаций эволюции возмущенной области возможно только при выполнении условия существования промежуточной асимптотики:

$$
\Pi_{01} \ll \Pi_{02}, \quad x_{01}(t) \ll x_{02}(t) .
$$

Использование такого модельного описания может претендовать только на выявление основных качественных закономерностей сложного процесса эволюции заряженной плазмы при выполнении условия сильной нелинейности.

Предельный переход эволюции в режиме торможения клинейному приближению задачи имеет вид

$$
\begin{gathered}
w \rightarrow Q \sqrt{\frac{T_{*}}{4 \pi t}}\left(1-n_{2} \frac{T_{*} x^{2}}{4 t}\right)^{\frac{1}{n}} \rightarrow Q \sqrt{\frac{T_{*}}{4 \pi t}} \exp \left(\frac{-T_{*} x^{2}}{4 t}\right), \\
T_{*}=T\left(n_{2}=0\right)=\text { const }
\end{gathered}
$$

осуществляется такой переход при выполнении условий

$$
n_{2} \ll 1 \quad \text { и } \quad\left|n_{2} \frac{T_{*} x^{2}}{4 t}\right|<1 .
$$

Такой переход к линейному приближению происходит при выполнении условия на эволюцию положения фронта:

$$
x_{0} \rightarrow \sqrt{\frac{4 t}{n_{2} T_{*}}},\left.\quad x_{0}\right|_{n_{2} \rightarrow 0} \rightarrow \infty .
$$

В линейном приближении отсутствует понятие „влияния“: возмущение асимптотически стремится к нулю на бесконечном расстоянии от источника. Следует отметить, что вещественное решение (17), соответствующее режиму торможения стримера, существует при любом положительном значении параметра $n$. В случае $n_{2} \ll 1$ использовать для описания эволюции заряженной плазмы идеализацию настоящей работы не следует, так как основными факторами будут эффекты, выходящие за рамки кинематического описания процесса. Вещественное решение (17), соответствующее режиму ускорения следует применять только при условии сильной нелинейности: $2>\left|n_{1}\right|>1$.

\section{Результаты и их обсуждение}

Отметим, что время длительности этапа ускорения $\Delta t_{1}$ фронта стримера, гораздо меньше времени длительности этапа торможения $\Delta t_{2}$ фронта. По этой причине размер стримера определяется этапом торможения. Введем понятие усредненной скорости перемещения фронта на основе определения

$$
\bar{V}_{0} \equiv \frac{x_{0}(t)}{t}
$$

Здесь $t$ - время прихода фронта в точку $x_{0}(t)$. Имеет место связь между скоростями

$$
V_{0}(t)=\frac{1}{n_{2}+2} \bar{V}_{0}(t)
$$


В нашей идеализации скорость фронта обращается в ноль только при значении $t=\infty$. В рамках использованной идеализации не происходит остановки возмущенной области на конечных расстояниях. В такой ситуации возможно введение различных критериев определения времени остановки возмущенной области и определения максимальной длины ее. Возможно определять эффект остановки следующим способом. За момент „остановки“ фронта $t=t_{\max }$ возьмем такой момент времени, когда скорость фронта $V_{0}\left(t_{\max }\right)$ примет некоторое малое фиксированное значение скорости $V_{\min }$. Величина $V_{\min }$ может задаваться с учетом точности фиксации скорости в эксперименте

$$
V_{0}\left(t_{\max }\right)=V_{\min } .
$$

Это соотношение позволяет определить $t_{\max }$. Значение максимальной длины $L$ в момент такой „остановки“ его имеет вид

$$
L=x_{0}\left(t_{\max }\right) .
$$

Экспериментально исследуемым параметром стримерно-лидерного процесса является скорость лидерной головки $V_{l}$. Процитируем высказывание [3]: „Продвижение в направлении понимания молниевых процессов не возможно без задания какой-то разумной зависимости лидерной скорости от внешних параметров“. Используется [1-3] понятие скорости стримера $V_{s}$. Между скоростью стримера $V_{s}$ и скоростью лидера $V_{l}$ установлено [13] приближенное соотношение

$$
V_{l} \approx V_{s} \Omega
$$

где параметр $\Omega$ - отношение времени распада плазмы (время прилипания электронов, либо время рекомбинации) к характерному времени увеличения газовой температуры. Для экспериментов в воздухе $\Omega \approx 10^{-1}$. В экспериментах со стометровыми искрами установлена эмпирическая зависимость [3]:

$$
V_{l}=a \sqrt{\left|U-U_{0}\right|}, \quad a=15 \mathrm{~ms}^{-1} \mathrm{~B}^{-\frac{1}{2}},
$$

где $U_{0}-$ внешний потенциал в месте нахождения головки лидера.

Локальная погонная емкость цилиндрического канала заряженной плазмы имеет логарифмическую зависимость от радиуса поперечного сечения канала, а зависимость от радиуса поперечного сечения канала для погонного сопротивления более сильная - степенная. По этой причине влияние сопротивления на проявление нелинейности более существенно, чем влияние емкости. Учитывая определение (4) и принимая во внимание слабое влияние емкости канала на нелинейные эффекты, можно допустить, что для стометровых искр имеет место пропорциональность $\left|U-U_{0}\right| \sim Q, V_{0} \sim V_{l} \sim \sqrt{Q}$.

Следствием закономерностей (14)-(16) появляется возможность определения параметра $n$ для ситуации сильной нелинейности: $n=2$. В таком случае имеет место закон убывания скорости фронта и закон увеличения длины стримера:

$$
\begin{aligned}
V_{0}(t) \sim t^{-3 / 4}, \quad V_{0}(t) & =\frac{1}{4} \bar{V}_{0}(t), \quad n=2, \\
x_{0}(t) & \propto t^{1 / 4} .
\end{aligned}
$$

Значение $n=2$ соответствует случаю достаточно сильной нелинейности. Это свидетельствует о некорректности использования линейных уравнений длинной линии с распределенными параметрами при описании эволюции стримера, порождаемого стометровой искрой. Для искр большей, чем сотни метров длины, нелинейность будет еще более сильной: $n>2$. А для искр, имеющих меньшую длину, в режиме торможения должно выполняться условие $0<n<2$.

Для нахождения плотности тока (3) и напряжения (4) нужны дополнительные сведения о функциях $R(w)$, $C(w)$. При получении оценок полей можно использовать приближение

$$
\begin{gathered}
C \approx \text { const, } \quad R \approx R_{1}\left(\frac{w}{w_{1}}\right)^{n}, \\
U \approx \frac{w}{C}, \quad j \approx-\frac{1}{C R_{1}}\left(\frac{w}{w_{1}}\right)^{n} \frac{\partial w}{\partial x} .
\end{gathered}
$$

Электрическое поле в таком приближении имеет представление

$$
\begin{aligned}
E(t, x)= & \frac{2 x Q^{\frac{2(n-1)}{n(n+2)}}}{C n(t \kappa)^{\frac{3}{n+2}}}\left[\frac{n}{2(n+1)(n+2)}\right]^{\frac{1}{n}} \\
& \times\left[\Pi_{0}^{2}-\Pi^{2}(t, x)\right]^{-\frac{1-n}{n}}, \quad x \leq x_{0}(t) .
\end{aligned}
$$

В случае $0<n<1$ электрическое поле имеет конечный скачок в точке $x=x_{0}(t)$ и имеется максимум при выполнении условия

$$
\frac{\partial E(t, x)}{\partial x}=0
$$

Существует критерий определения максимальной длины стримера [2], отличный от способа (18). Экспериментально измеряемой величиной является так называемое „критическое поле“ $E_{\min }$ [2]. Это предельно слабое поле, когда фронт стримера еще способен передвигаться: $V_{0}(t) \rightarrow V_{0}\left(t_{\max }\right)=V_{\min }$.

Существует рекомендация, основанная на усредненных лабораторных измерениях в воздухе:

$$
E_{\min }=465 \frac{\mathrm{kV}}{\mathrm{m}} \text {. }
$$

В случае $0<n<1$ можно определить параметры $L$ и $t_{\max }$ из системы двух уравнений

$$
\begin{gathered}
E_{\min }=E\left(t_{\max }, L\right), \\
\left.\frac{\partial E(t, x)}{\partial x}\right|_{x=L, t=t_{\max }}=0 .
\end{gathered}
$$

Журнал технической физики, 2018, том 88, вып. 3 


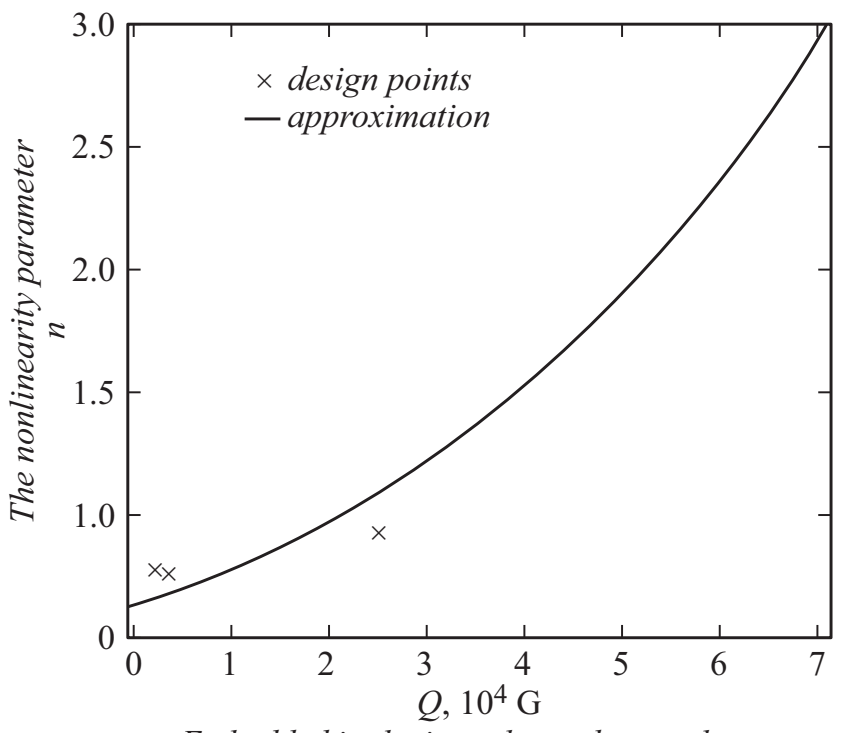

Embedded in the interelectrode gap charge

Зависимость параметра нелинейности $n(Q)$ на этапе торможения стримера. $Q$ - величина заряда внедряемого в межэлектродный промежуток. Крестиком отмечены данные экспериментов [12-17].

В случае „очень коротких“ стримеров, когда $n \ll 1$, использовать кинематическое приближение (5)-(8) не следует, так как в такой ситуации основным влияющим фактором являются эффекты линейного приближения, которые не учитываются в используемой идеализации.

При значениях $n>1$, структура поля $E(t, x)$ существенно иная: в точке $x=x_{0}(t)$ имеется бесконечный скачок электрического поля. В такой ситуации в рамках используемой модели остановку стримера нельзя определять на основе использования понятия $E_{\min }$. Для этой цели можно использовать критерий (18).

Следует отметить, что сформулированная на основе (10)-(17) автомодельная задача имеет четыре размерных определяющих параметра: $t, x, Q, \kappa$ и один безразмерный параметр $n$. Безразмерный параметр нелинейности $n$ задается в такой задаче независимо от размерных параметров: $Q, \kappa$. Анализ [21] лабораторных экспериментальных данных о стримерах [12-17] на стадии торможения показывает, что существует взаимная связь между параметром нелинейности $n=n_{2}$ и внедряемым в межэлектродный промежуток зарядом $Q$. Эта связь описывается следующей аппроксимирующей функцией [21]:

$$
n(Q)=0.64 \cdot \exp [22 \cdot Q] .
$$

Закономерность (19) изображена на рисунке крестиком отмечены данные экспериментов.

\section{Заключение}

1. Предложенная выше одномерная нелинейная кинематическая модель заряженной плазмы основана на законе сохранения суммарного заряда $Q$. Эта модель является очень упрощенной, „жесткой“ идеализацией реального процесса. Многие достаточно сложные физические процессы при этом формально не учитываются в явном виде. Однако следует отметить, что значительная часть сильно нелинейных процессов в предложенной модели принимается во внимание фиксацией одного безразмерного параметра $n$, который может основываться на данных экспериментов. Последнее утверждение подтверждается сопоставлением аналитических формул с результатами экспериментов [12-18].

2. Перечислим преимущества одномерного автомодельного решения (14), (15), (17) по сравнению с решениями, предложенными ранее другими авторами. Для описания пространственно-временных стадий ускорения и торможения стримера длинной искры получены простые аналитические формулы. Установлена связь скорости фронта с определяющими параметрами задачи. Аналитические формулы правильно описывают основные качественные закономерности процесса. Это подтверждается данными экспериментов. Для этапа торможения это сделано при значениях нелинейного параметра $3 \geq n \geq 0.77$ и при изменении внедряемого в межэлектродный промежуток заряда $Q$ от значения $Q=$ $=3.6 \cdot 10^{-5}$ до значения $Q=7.1 \cdot 10^{-4}$.

3. Получено представление для максимальной длины стримера.

4. Отмечены аномальные свойства автомодельного режима ускорения фронта заряженной плазмы.

5. Качественные закономерности эволюции возмущенной области описываются кинематическим приближением (5)-(8) только в случае сильной нелинейности: $|n| \geq O(1)$. В случае „очень коротких“ стримеров, когда $n \ll 1$, использовать кинематическое приближение (5)-(8) не следует.

\section{Список литературы}

[1] Базелян Э.М., Райзер Ю.П. // УФН. 2000. Т. 170. № 7. C. $753-769$.

[2] Базелян Э.М., Райзер Ю.П. Физика молнии и молниезащиты. М: Физматлит, 2001. Гл. 2. 320 с.

[3] Базелян Э.М., Райзер Ю.П. Искровой разряд. М: Изд-во МФТИ, 1997. 287 с.

[4] Яковленко С.И. // ЖТФ. 2004. Т. 74. Вып. 9. С. 47-54.

[5] Лозанский Э.Д., Фирсов О.Б. Теория искры. М: Атомиздат. 1975. Гл. 6-7.

[6] Vitello P.A., Penetrante B.M., Bartsley J.N. // Phys. Rev. E. 1994. Vol. 49. N 4. P. 5574-5598.

[7] Aleksandrov N.L., Bazelyan E.M. // J. Phys. D: Appl. Phys. 1996. Vol. 29. P. 740-752.

[8] Kulikovsky A.A. // Phys. Rev. E. 1998. Vol. 57. N 6. P. 7066-7074.

[9] Babaeva N.Y., Naidis G.V. // J. Phys. D: Appl. Phys. 1996. Vol. 29. P. 2423-2431.

[10] Бабаева Н.Ю., Найдис Г.В. // Письма в ЖТФ. 1999. Т. 25. Вып. 3. С. 19-27. 
[11] Arrayas M., Erbert U., Hundsdorfer W. // Phys. Rev. Lett. 2002. Vol. 7. P. $174-502$.

[12] Gallimberti I. // J. Phys. D: Appl. Phys. 1972. Vol. 5. P. 2179-2189.

[13] Schneider K.N. // Electra. 1977. N 53. P. 31-153.

[14] Goelian N., Lalande P., Bondiou-Clergerie A., Bacchiega G.L. // J. Phys. D: Appl. Phys. 1997. Vol. 30. P. 2441-2452.

[15] Schneider K.N. // IEE Proc. A. 1986. Vol. 133 (7). P. 3-153.

[16] Bondiou A., Gallimberti I. // J. Phys. D: Appl. Phys. 1994. Vol. 27. P. $1252-1266$.

[17] Ortega P. // Proc. High Voltage Engineering: 7th ISH Intern. Sympos. 1991, Dresden. Papers and summaries. P. 105-108. Ed.: Kleber W. Dresden University, 1991.

[18] Won J.Yi., Williams P.F. // J. Phys. D: Appl. Phys. 1972 Vol. 35. P. 205-218.

[19] Whitham G.B. // Proc. Roy. Soc. A. 1950. Vol. 203. P. 571-581.

[20] Уизем Джс. Линейные и нелинейные волны. М.: Мир, 1977. 622 c. (Whitham G.B. Linear and nonlinear waves. NY., 1974.)

[21] Павлов В.А. // Прикладная механика и техническая физика. 2010. Т. 51 № 6. С. 1-11.

[22] Зельдович Я.Б., Компанееи, А.С. К теории распространения тепла при теплопроводности, зависящей от температуры. В сборнике, посвященном 70-летию акад. А.Ф. Иоффе. М.: изд-во АН СССР, 1950. С. 61-71.

[23] Баренблатm Г.И. // Прикл. мат. мех. 1952. Т. 16. № 31. C. $67-78$.

[24] Баренблатm Г.И. Подобие, автомодельность, промежуточная асимптотика. Л.: Гидрометеоиздат, 1978. Гл. 2. $207 \mathrm{c}$. 